\title{
SOFT X-RAYS INDUCED FRAGMENTATION OF CORE EXCITED POLYATOMIC MOLECULES
}

\author{
M.-J. HUBIN-FRANSKIN, $\dagger$ M. FURLAN, J. DELWICHE $\dagger$ \\ and $\mathrm{H}$. AOUNI
}

Laboratoire de Spectroscopie d'Electrons Diffusés, Institute de Chimie B6, Université de Liège, Sart Tilman par 4000 Liege 1, Belgium

Recent progress made in the soft X-ray induced fragmentation of polyatomic molecules has been briefly reviewed. The Coulomb explosion model has been shown to be less valid as the size of the molecule increases.

\section{INTRODUCTION}

When an isolated molecule absorbs soft X-rays a core electron may be promoted into an unoccupied molecular orbital (valence, Rydberg) or into the continuum. The excited system decays by the emission of one or more Auger electrons and formation of a singly or multiply charged molecular ion which generally dissociates into ionic fragments and possibly neutral(s). The core hole decay channels of a core excited molecule have gained interest quite recently. This has been partly due to the availability of the synchrotron radiation as a tunable photon source covering the whole UV to soft X-ray range and to sophisticated detection methods of multiply charged particles coupled to photoionisation. In addition, it has required progress in the spectroscopy itself of the core excited states and of the mechanism of core electron excitation (single electron, multielectron, shape resonance). This field is now being developed thanks to the improvement of high resolution monochromators for the soft X-ray range and thus of high resolution inner shell photoabsorption and photoionisation with resolution as high as $40 \mathrm{meV}$ at the $1 \mathrm{~s}$ carbon edge. ${ }^{1}$

In our laboratory special effort is being made to contribute to the high resolution inner shell photoabsorption by the $(e, e)$ Dipolar Spectroscopy which makes use of an electron beam and allows pseudophotoabsorption when operating in pseudo-optical conditions. ${ }^{2}$ The method has been the Electron Energy Loss Spectroscopy. Preliminary results are presented for nitrogen and hexafluorobenzene.

The dynamics of core excited molecules will be discussed in terms of the Coulomb explosion model and its validity for large polyatomic systems and also of the

† Maître de Recherches du FNRS. 
localisation of two valence holes on the same atomic site of the core hole. These effects will be illustrated for methylamine and hexafluorobenzene after excitation of a $1 \mathrm{~s}$ core electron. The method has been photoionisation using the synchrotron radiation, time-of-flight mass spectrometry and charged particles multicoincidence detection.

\section{EXPERIMENTAL PART}

\section{The Electron Energy Loss Spectrometer}

The new Electron Energy Loss Spectrometer has been a VSW instrument equipped with two large radius electrostatic $180^{\circ}$ selectors, one operating as an electron monochromator and the second as an electron analyser. Beam transport and focusing have been performed by a multielement electron optics. The instrument has been designed to work at $0^{\circ}$ scattering angle. The impact energy is tunable between 1 and $5 \mathrm{KeV}$.

The molecular excitation spectrum has been obtained by scanning the residual kinetic energy (K.E.resid.) of the electrons after being scattered inelastically by the molecular target. This K.E. resid. is directly related to the energy loss $\left(E_{\text {loss }}\right)$ of the incident electrons:

$$
\text { K.E.resid. }=E_{i}-E_{\mathrm{loss}}=E_{i}-E^{*}
$$

$E^{*}$ is the excitation energy of the molecular target, $E_{i}$ the impact energy.

When operating in pseudo-optical conditions (low angular momentum transfer, low scattering angle) the electron energy loss spectra are quite similar to the photoabsorption ones.

\section{The Photoionisation Experiment}

The photoionisation experiment has been based on the analysis of the ions (parent, fragment, singly or multiply charged) according to their mass or to their flight times to reach a detector. It has made use of the detection in coincidence of charged particles which are formed in the same ionisation event by the PhotoElectron PhotoIon Photolon CO/incidence (PEPIPICO) method. The spectrometer and detection system have been described previously. ${ }^{3,4}$ The photon source has been the synchrotron radiation of SuperACO of LURE (Orsay, France).

\section{RESULTS}

\section{Inner Shell Spectroscopy}

The K shell nitrogen molecule excitation spectrum recorded with the electron energy loss spectrometer is displayed in Figure 1. It has been obtained by inelastic electron scattering in the forward direction at $2 \mathrm{KeV}$ incident energy and with a $0.4 \mathrm{eV}$ energy 


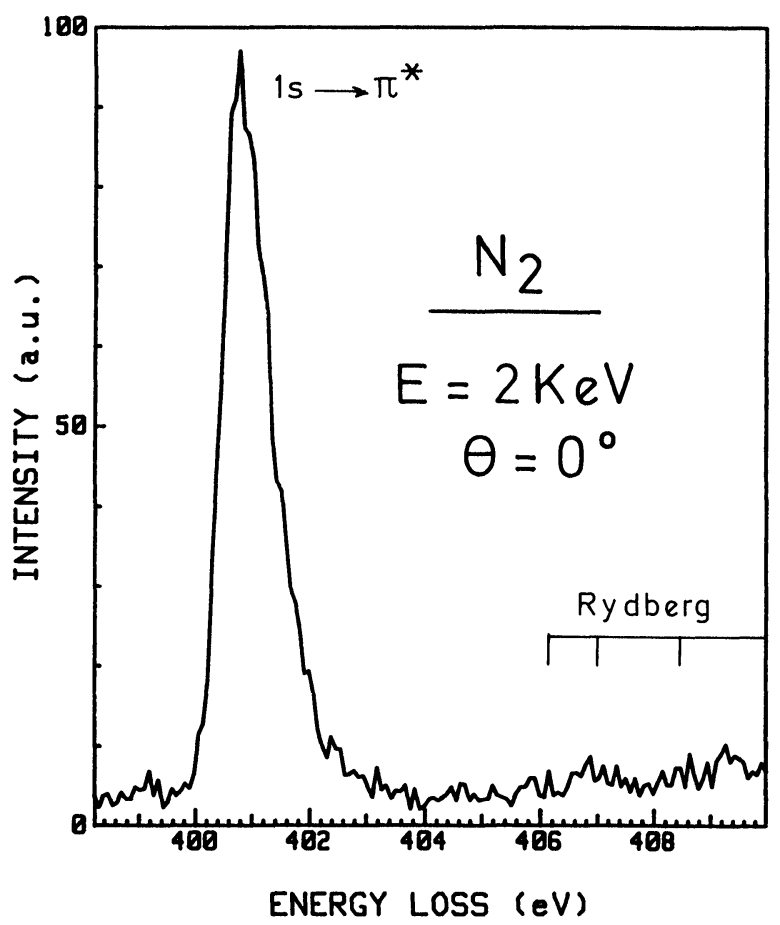

Figure 1 Inner shell electron energy loss spectrum of the nitrogen molecule. The energy resolution is $0.4 \mathrm{eV}$.

resolution. It has been limited to the excitation region below the ionisation edge. It is dominated by the discrete resonance to the lowest energy unoccupied valence orbital $\pi_{g}^{*}$. At higher energy the transitions to Rydberg series are observed with a quite lower intensity. At higher resolution $(0.13 \mathrm{eV})$ three and two terms of the $s \sigma$ and $p \pi$ series respectively have been observed as shown in Figure 2a. In addition, the vibrational progression of the $1 \mathrm{~s} \rightarrow \pi_{g}^{*}$ band has been partly resolved as shown in Figure $2 \mathrm{~b}$. The frequency of $0.230 \mathrm{eV}$ is in excellent agreement with the literature data. ${ }^{2,5}$

The $\mathrm{K}$ shell hexafluorobenzene excitation spectra are displayed in Figures $3 \mathrm{a}$ and $3 \mathrm{~b}$ at the $\mathrm{C} 1 \mathrm{~s}$ and F1s edges, respectively. They have been limited to the lowest energy antibonding valence $\sigma^{*}$ and $\pi^{*}$ molecular orbital excitations.

The C1s spectrum recorded with $0.35 \mathrm{eV}$ resolution is dominated by the $1 \mathrm{~s}_{c} \rightarrow 1 \pi^{*}$ band which is completely resolved from the $2 \pi^{*}$ one. In addition definitely there is a transition at $289 \mathrm{eV}$ suggested previously to involve a $\sigma_{\mathrm{C}-\mathrm{F}}^{*}$ orbital. ${ }^{6}$

The F1s spectrum recorded with $0.2 \mathrm{eV}$ resolution is also dominated by excitation of the $1 \pi^{*}$ molecular orbital, the band remaining quite broad despite the resolution higher than in previous studies. ${ }^{6}$

By Inverse Photoemission Spectroscopy (IPS) ${ }^{7}$ the lowest unoccupied molecular 

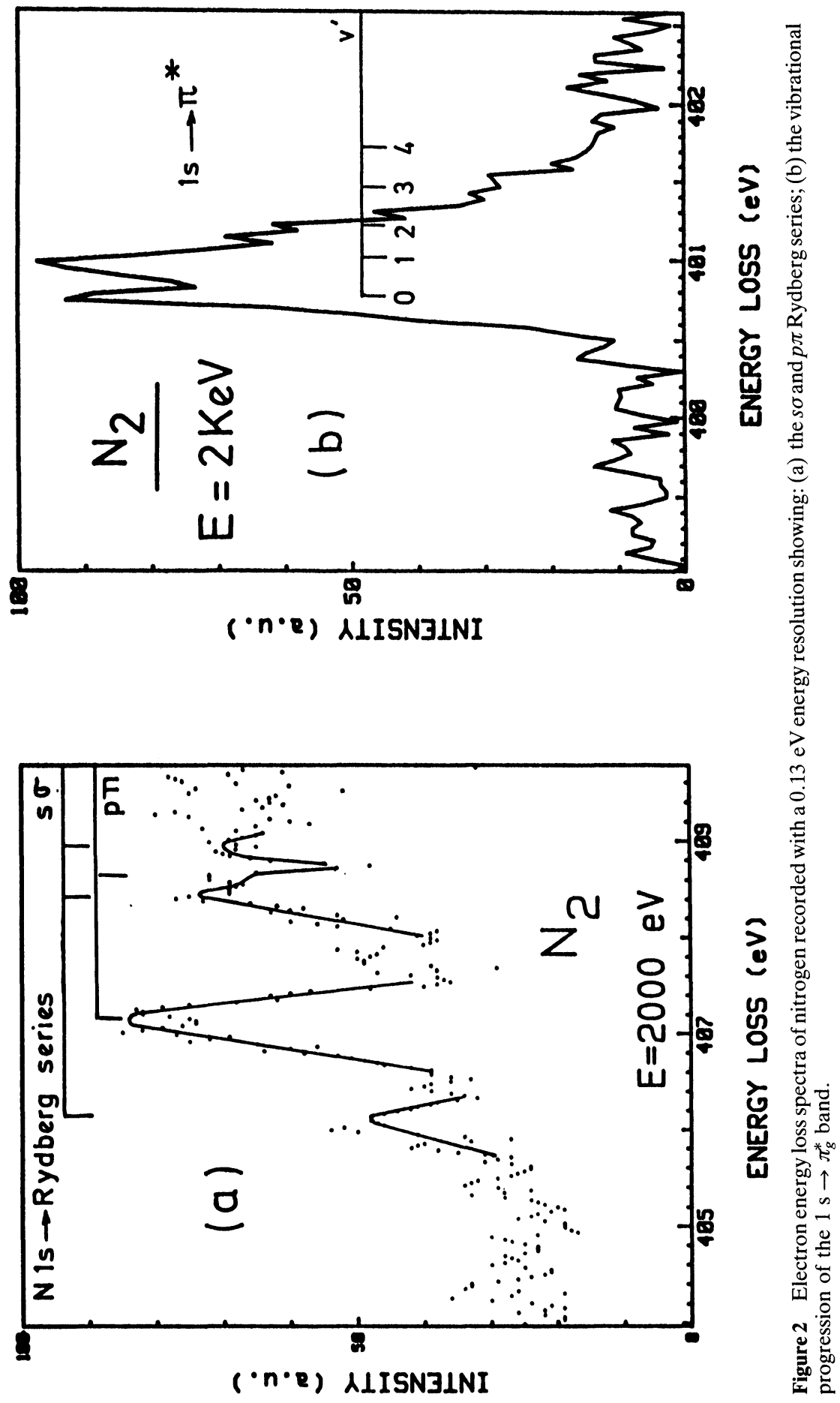


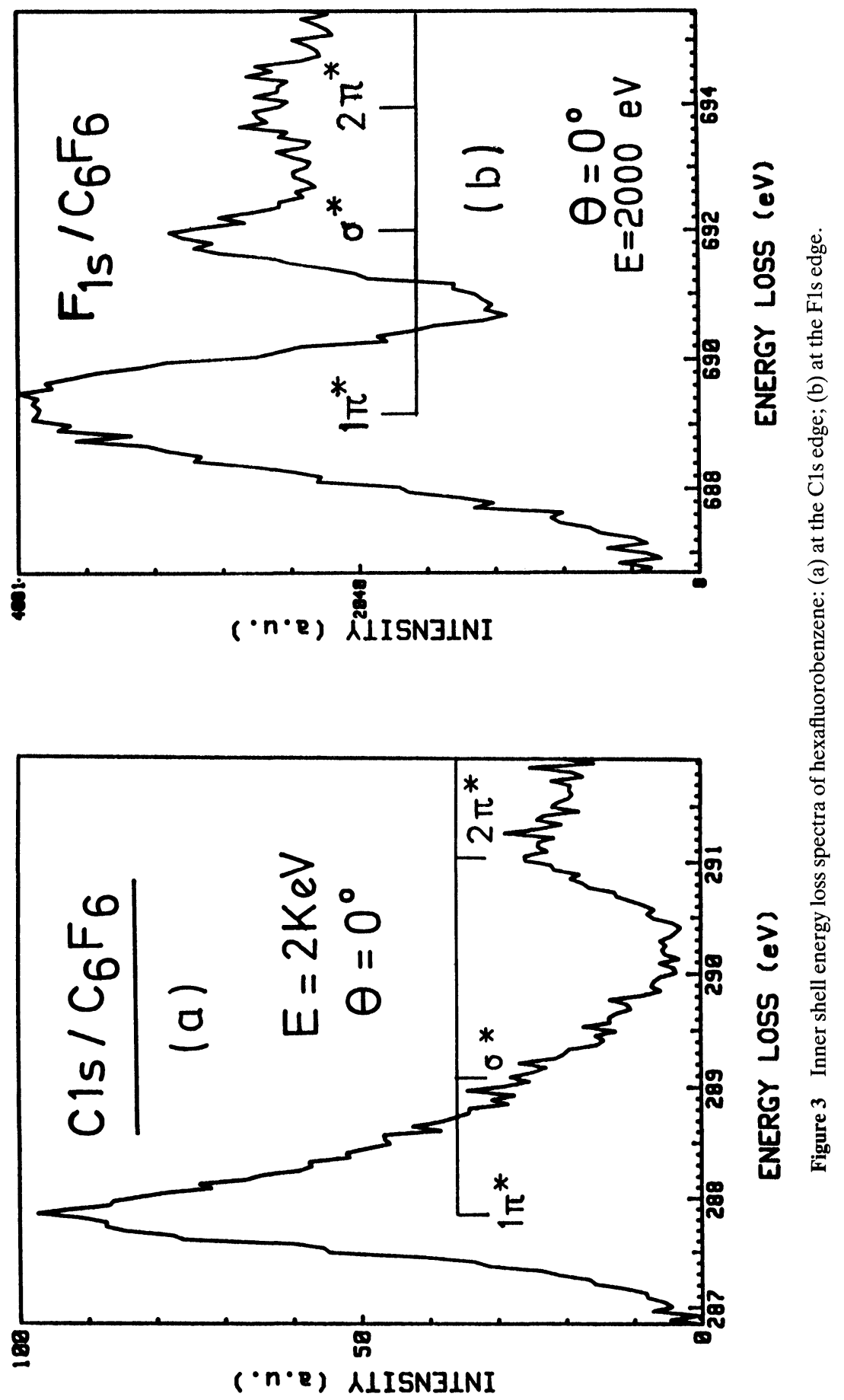


orbital has been suggested to be a $\sigma^{*}$ one. However, it cannot be resolved in our experiments even with the improved resolution. Very likely when a $1 \mathrm{~s} \mathrm{C}$ electron is excited, the $\sigma^{*}$ final state is shifted below $1 \pi^{*}$ less than in IPS and in the $1 \mathrm{sF}$ core electron spectrum. This reduced shift (less than $1 \mathrm{eV}$ ) and the expected low intensity compared to the excitation of $1 \pi^{*}$ might make it difficult to be resolved from the $1 \pi^{*}$ band.

At the present stage of testing resolution these performances do not represent the limit of the spectrometer. In addition a multidetector incorporating microchannel plates, a phosphor screen, fiber optics and a photodiode array with charge coupled device will be installed quite soon to improve the sensitivity.

The great advantage of the $(e, e)$ Dipolar Spectroscopy is that resolution can be kept constant in quite a broad excitation energy range.

\section{Inner Shell Excited States Fragmentation}

Relaxation of inner shell excited molecules generally proceeds by emission of one or more Auger electron(s) and the formation of a singly or multiply charged molecular ion which generally dissociates into ionic fragments and possibly neutral(s). The fragmentation of multiply charged ions may be ruled by Coulomb explosion due to the repulsion between the positive charges but also may proceed in multistep by charge separation and deferred charge separation. ${ }^{8}$ Sometimes two valence holes remain localised on the same atomic site of the core hole. This phenomenon called Coulomb localisation is indicative that a localised picture of bonding may be more appropriate for describing the electronic and dissociation channels than a molecular orbital picture. ${ }^{9}$

For diatomics about $95 \%$ of the dissociation leads to atomic ions with large amount of kinetic energies. These latter are around $7 \mathrm{eV}$ for singly charged ions and about twice for doubly charged ions. ${ }^{9}$ Thus, the Coulomb explosion model appears to be relevant. Recent studies of time-of-flight mass spectra of two larger polyatomic systems ${ }^{10,11}$ has shown up that the Coulomb explosion model is lesser valid as the size of the molecule increases. Indeed, the yield of atomic ions produced by relaxation of the $1 \mathrm{~s}$ nitrogen core hole in methylamine (seven atoms) has been measured to be about $52 \% .{ }^{10}$ This amount is lower than for diatomics and for triatomics $(75 \%$ for $\mathrm{N}_{2} \mathrm{O}$ ). ${ }^{12}$ For hexafluorobenzene (12 atoms) the yield of atomic ions drops to $32 \%{ }^{11}$ when a $1 \mathrm{~s}$ fluorine or a $1 \mathrm{~s}$ carbon core electron is excited.

The doubly charged atomic ion $\mathrm{F}^{2+}$ is formed when a $1 \mathrm{~s}$ fluorine electron of hexafluorobenzene is excited. It corresponds to the localisation of the two valence holes on the same atomic site. Such ions have also been reported in $\mathrm{SiF}_{4}$ but not in $\mathrm{CF}_{4}{ }^{13}$ These observations are consistent with the weaker intramolecular interactions in larger polyatomic systems and thus larger $\mathrm{F}-\mathrm{F}$ distances.

\section{CONCLUSIONS}

High resolution and high sensitivity inner shell photoabsorption or pseudophotoabsorption by $(e, e)$ Dipolar Spectroscopy will largely contribute to test the validity of 
the Equivalent Core model and of the core hole localisation picture and to enlighten the core electron excitation mechanism (single electron, multielectronic, shape resonance). In addition, for larger polyatomic molecules it is a unique tool for the spectroscopy of the LUMO.

The fragmentation of large polyatomic systems after $\mathrm{K}$ shell excitation becomes dominated by multistep processes as the size of the molecule increases.

\section{Acknowledgements}

We are grateful to the Fonds National de la Recherche Scientifique of Belgium for research positions (J. D. and M.-J. H.-F.) and financial support. We also acknowledge the Patrimoine of the University of Liège and the Service de la Programmation de la Politique Scientifique for financial support. We also thank the NATO (contract 870484), the staff of the LURE and of the LINAC (Orsay, France).

\section{References}

1. C. T. Chen, Y. Ma and F. Sette, Phys. Rev. A. 40, 6737 (1989).

2. R. N. S. Sodhi and C. E. Brion, J. Electr. Spectr. Rel. Phen. 34, 363 (1984).

3. T. LeBrun, M. Lavollée and P. Morin, X90 Conference, Knoxville USA (1990).

4. M. Simon, M. Lavollée, T. LeBrun and P. Morin, Nucl. Instr. A. (in press).

5. G. King, F. H. Read and M. Tronc, Chem. Phys. Lett. 52, 50 (1977).

6. A. P. Hitchcock, F. Fisher, A. Gedanken and M. B. Robin, J. Phys. Chem. 91, 531 (1987).

7. R. Dudde, B. Reihl and A. Otto, J. Chem. Phys. 92, 3930 (1990).

8. D. A. Hagan and J. H. D. Eland, Rapid Communication in Mass Spectrom. 3, 186 (1989).

9. D. M. Hanson, Adv. Chem. Phys. 77, 1 (1990), ed. I. Prigogine, S. Rice, John Wiley \& Sons.

10. R. Thissen, M.-J. Hubin-Franskin, J. Delwiche, H. M. Simon and M. Lavollée, to be published.

11. K. Ibrahim, M.-J. Hubin-Franskin, M. Furlan, J. Delwiche, M. Simon and M. Lavollée, to be published.

12. J. Murakami, M. C. Nelson, S. L. Anderson and D. M. Hanson, J. Chem. Phys. 85, 5755 (1986).

13. D. A. Lapiano-Smith, C. I. Ma, K. T. Wu and D. M. Hanson, J. Chem. Phys. 90, 2162 (1989). 